\title{
Research on Symbolization of Domestic Yogurt Packaging
}

\author{
Yang Du, a , Zongyuan Bian ${ }^{2, b}$ and Xia Geng ${ }^{3, c}$ \\ ${ }^{1,2,3}$ School of Architecture and Art Design, Beijing Jiaotong University, Beijing 100044, China \\ a807249775@qq.com, b799690386@qq.com, '850271255@qq.com
}

\begin{abstract}
As an indispensable nutrition in people's lives, liquid milk occupies its own place in the market. In recent years, healthy diet is advocated, and yogurt has attracted much concern because it can help people reduce weight and contribute to better sleep. In this paper, element extraction and cross analysis are used to study the main visual effect of food packaging. Adjacent axis and pedicle axis are used to classify the yogurt boxes, and part of them are extracted for in-depth analysis so as to know the correlative relationship between different elements in yogurt box packaging design and give a prospect of yogurt box packaging design in the future based on this.
\end{abstract}

Keywords: Yogurt box packaging; Symbol; Cross analysis; Design rule

\section{国内酸奶包装符号化研究}

\author{
杜洋 ${ }^{1}$, 边宗圆 ${ }^{2}$, 耿霞 ${ }^{3}$
}

1.2.3. 北京交通大学建筑与艺术学院, 北京市, 100044

摘要: 液态奶作为目前人们生活中不可或缺的营养品, 在市场上占有自己的一席之地, 近年来提倡健康饮食, 酸奶由于 其减肥, 助眠等功效, 备受关注。文章通过元素提取和交叉分析法对食品包装的主视觉进行研究, 利用毗邻轴和系谱轴对酸 奶盒进行分类, 同时从中提取一部分进行深入剖析, 管窥酸奶盒包装设计中不同要素之间的关联性规律, 并以此为依据进行 未来酸奶盒包装设计展望。

关键词: 酸奶盒包装; 符号; 交叉分析; 设计规律

中图分类号：J524.2 文献标志码, $\mathrm{A}$

引言

近年来, 国内乳品行业中酸奶的发展引人注目, 酸奶包装是在消费者购买前最直接的表现品质的媒介, 也是吸引消费者购买的重要环节。酸奶盒包装日益呈现出多样化发展的趋势。酸奶盒包装主要由品牌名、 主视觉、诉求点三部分组成, 这里根据价格将酸奶定位成高中低端, 并针对其中的高端酸奶盒进行切分和 比较, 探究出高端酸奶盒之间存在的共同点, 合理预测未来酸奶盒包装设计发展。

\section{1 国内酸奶盒包装整体分析}

对国内线上和线下的酸奶进行搜集后, 根据价格分区, 将酸奶定位成高中低端, 低端 0-2.4 元, 中端 2. 5-3.5 元, 高端 3.6-12 元（以上价格分区按每 100g 的酸奶价格界定）（图 1）。将酸奶盒分区进行颜 色、内容、突出元素的粗略比较, 可以得出以下结论: 


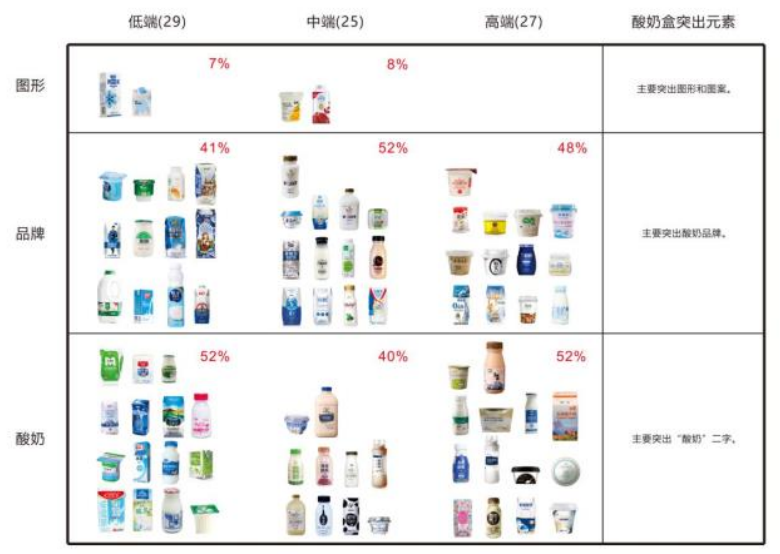

图 1 高中低端酸奶盒样品展示

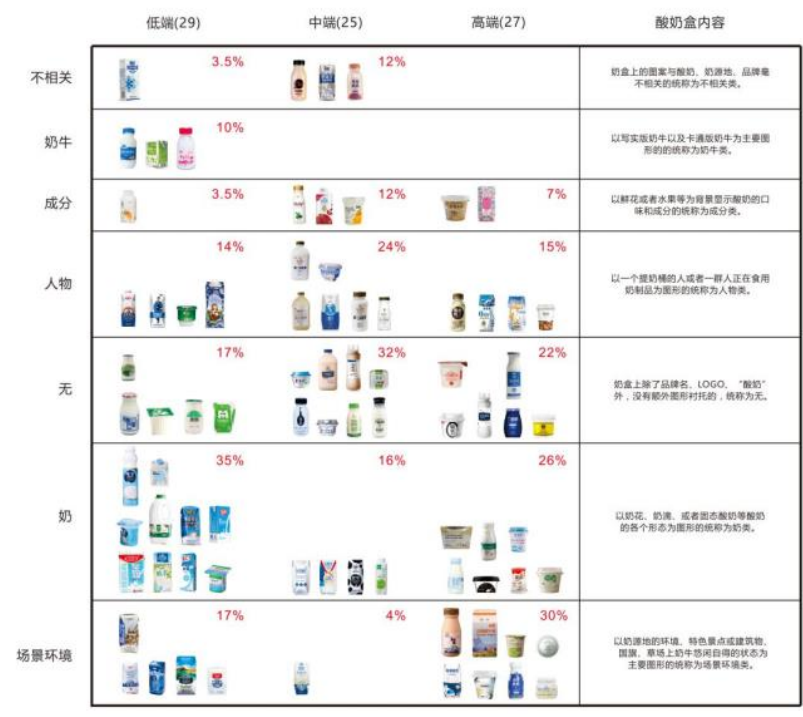

图 3 酸奶盒内容比较

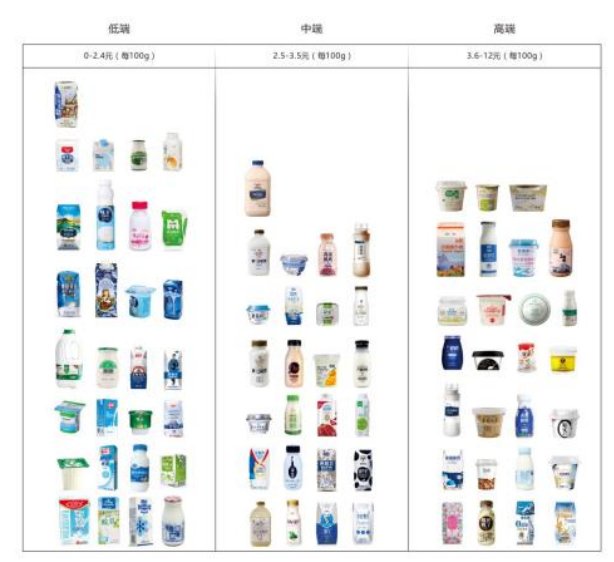

图 2 酸奶盒突出元素比较

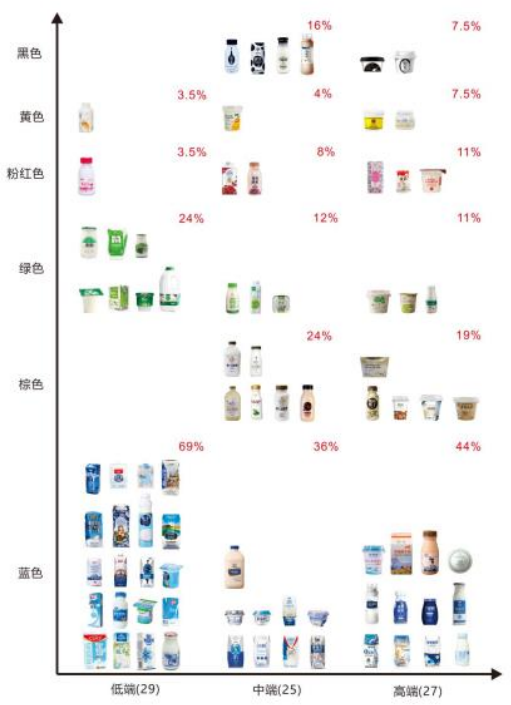

图 4 酸奶盒颜色比较

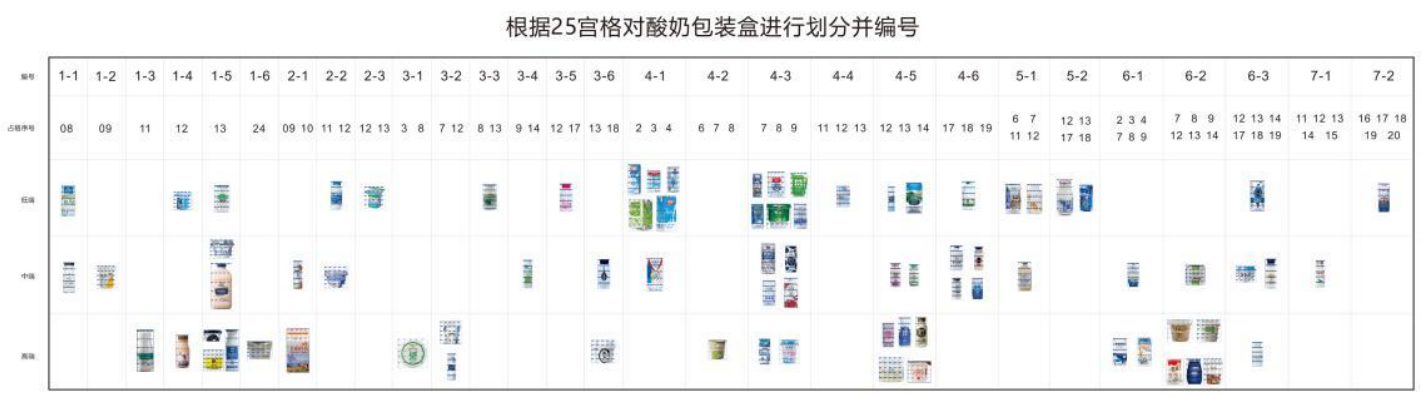

图 5 品牌位置比较

在突出元素上, 低端酸奶盒突出 “酸奶” 告诉消费者产品本质; 中端和高端酸奶盒大部分是突出品牌, 依靠品牌效应（图 2)。在内容上, 低端酸奶盒图案元素混杂, 有许多不相关的图案, 颜色鲜亮, 种类繁 多。高端酸奶盒图案占比小或做为底纹出现, 不是第一视觉重点（图 3)。在颜色上, 低端酸奶盒大篇幅 使用蓝色或绿色, 颜色混杂, 纯度高。中端和高端酸奶盒上的颜色多为两种, 多留白 (图 4)。在品牌位 置上, 低端酸奶盒大多把 “酸奶” 二字放在居中位置, 品牌名放在周边位置, 高端酸奶品牌名大部分在奶 盒的中间位置（图 5）。 


\section{2 对高端酸奶盒深入研究}

高端酸奶因其定位人群高端, 在包装上规整大气，容易找出其包装上的规律。文章中将高端酸奶盒单 独提出, 从颜色、品牌位置、内容、质量等多方面进行深入分析，发掘规律。

\section{1 颜色提取结合品牌位置和元素进行分析}

通过搜集，共找到 27 个高端酸奶样本（图 6)，并对样本除白色外的主要颜色进行提取，颜色分布 如下:

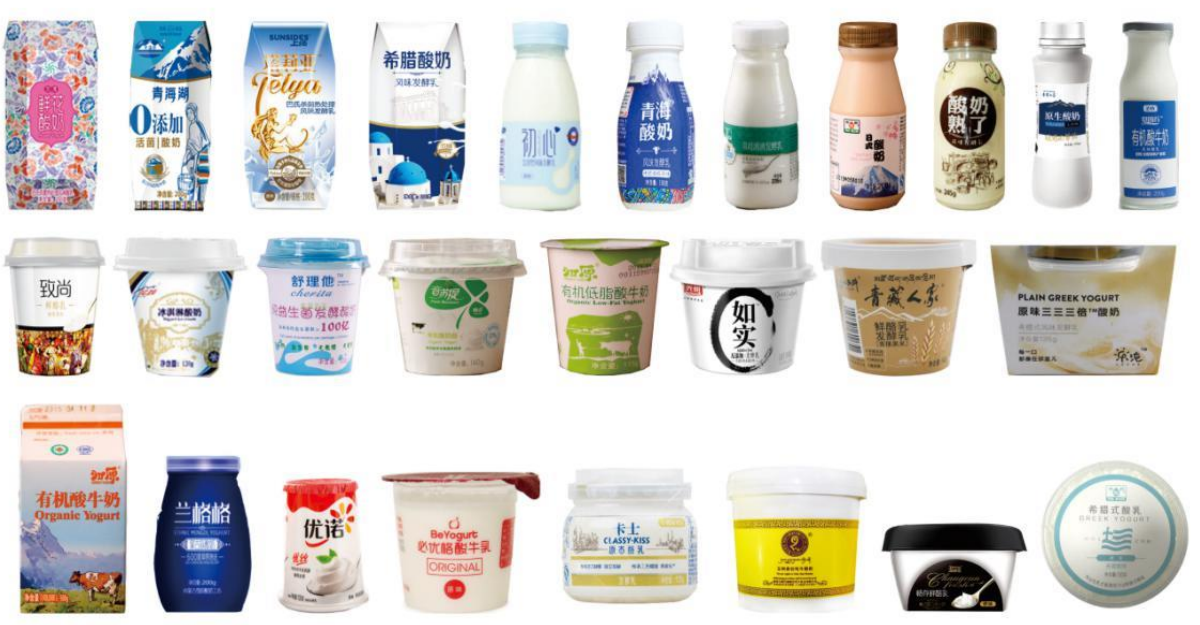

图 6 高端酸奶盒样品展示

蓝色占据主要地位, 但相较于低端酸奶盒中蓝色占比已大幅降低, 同时增加了几种颜色, 这些常用的 颜色在使用上都降低了纯度和亮度。将酸奶盒的颜色与品牌位置、元素等进行交叉分析, 以找出酸奶盒的 颜色和其他方面的规律。

大多数瓶身是蓝色, 品牌位置居中的, 突出的都是品牌。蓝色在瓶身上多作为底色出现, 蓝色给人一 种后退, 收缩感, 作为底色更突显品牌名 ${ }^{[1]}$ 。蓝色是冷色调, 所以瓶身使用蓝色的, 奶源地多为高原或者 较寒冷地区。蓝色、绿色等容易给人一种酸味的味觉刺激, 看到蓝色包装盒, 首先就会想到酸奶 ${ }^{[2]}$ 。

在 25 宫格中, 13 是视觉中心, 由于品牌名的大小不同，占格多少也有所不同，一般品牌名长度占据 三格, 分别是 12、13、14, 再根据字体高矮, 向上或向下延伸一行。“兰格格” 酸奶瓶身是椭球形, 品牌 位置靠下会处于视觉盲区, 所以向上提高了品牌名位置。

所有瓶身主颜色是红色的品牌位置都居中, 突出的元素都是 “酸奶”。红色本身就给人一种前进感, 所以在文字上使用红色更为突出。同时红色是暖色, 通常用作奶源地或者该品牌企业处于南方或温暖地区。 白色和红色也会给人一种甜味的味觉刺激, 隐喻该酸奶口味偏甜。利用同样的分析方法对于其他颜色的酸 奶盒进行分析, 同样可以得出结论。

\section{2 奶盒正面内容结合多方面进行分析}

归原酸奶在包装上着重突显奶源地的环境, 利用远处湛蓝的天空和映照下的白蓝色山峰为远景, 构成 奶盒上淡蓝色的主色调, 近处是碧绿的草地和悠闲的奶牛。奶源地的环境体现了酸奶高昂的品质, 所以在 包装上着重突出 “酸奶” 二字, 奶盒上的图案会使看到包装的人下意识相信这个酸奶是高端的。除可以利 用奶源地环境突显酸奶品质外, 瓶身上什么元素都没有的以及奶类、人物类、成分类, 只能将图案淡化, 主打品牌，品牌名位置基本都居中。 


\section{3 酸奶的重量结合材质等进行分析}

在 27 个样本中, $100 \mathrm{~g}-200 \mathrm{~g}$ 之间的酸奶占到了样本的一半, $200 \mathrm{~g}$ 的占到了样本的四分之一, 说明重 量小于等于 $200 \mathrm{~g}$ 的包装是主流，用过即抛的便利包装最受消费者欢迎。

\section{3 总结}

在酸奶盒包装中，正视图共包含品牌名、主视觉、诉求点三大方面，体现在具体元素上就是颜色、品 牌位置、内容、形状等，对这些元素进行细分和深入研究找寻规律，对未来的食品包装设计有重大的理论 及实践意义。经过多个样本分析, 总结出以下几条规律：1、绝大多数的品牌名都在包装的居中位置。2、 高端酸奶盒主色调为白色, 每个奶盒上的颜色种类少, 纯度低。3、酸奶重量在 $100 \mathrm{~g}-200 \mathrm{~g}$ 之间 (包括 $200 \mathrm{~g}$ ) 的占据主流，能够一次性喝完的小容量，用过即抛的包装最受欢迎。4、所有可以重复开合的圆柱形酸奶 盒都采用塑料瓶，一次性包装多采用纸盒。

未来的高端酸奶盒包装设计可能在色彩上更加丰富多彩, 底纹颜色淡化, 主体文字颜色突出, 在推出 酸奶时, 主打品牌。酸奶的品牌或者品名有统一的规格, 位于酸奶包装的正中央, 并根据奶盒形状进行上 下左右的微调。酸奶的重量在 $200 \mathrm{~g}$ 左右。

\section{参考文献}

[1] 张佳宁，张瑾．食品包装设计与色彩心理作用．包装世界，2008. 09. 92-94.

[2] 何进媚. 就色彩心理学谈食品包装设计. 桂林理工大学高等职业技术学院，2013-09-66-2.

[3] 赵盈盈. 人性化包装设计探究. 齐鲁工业大学, 2013.6.8.

[4] 穆亚君．西湖龙井茶包装设计研究．杭州师范大学，2013.10.8.

[5] 王佳慧. 论包装设计的延伸功能. 西南交通大学, 2012.9. 29.

［6］林月华，姜玉，吴艳叶. 网购包装与传统包装差异研究. 包装世界，2016.6.10-12.

[7] 廖雨瑶, 陈丹青, 李伟等. 智能包装研究及应用进展. 绿色包装, 2016. 10, 39-46.

[8］许文凯．智能包装的新宠一RFID 技术 $[J]$. 印刷 杂志, 2013，(02):57-59.

[9] Hill SC, Htet Y, Kauffman J, Han IY, Dawson PL, Pennington WT, Hanks TW. Polydiacetylene-based smart packaging [J]. Phys Meth Food Anal: Am Chem Soc, 2013, 1138: 137 - 54.

[10] Verghese K. Crossin E, JoHands M. Packaging Materials [M]. Pack- aging for Sustainability. Springer London: 2012.

\section{References:}

[1] Zhang Jianing, Zhang Jin. Food Packaging Design and Color's Psychological Effects. Packaging World, 2008.09.92-94.

[2] He Jin-mei. Design of Food Packaging in Color Psychology. College of Higher Vocational and Technical Education, Guilin University of Technology, 2013-09-66-2.

[3] Zhao Yingying. Research on Humanized Packaging Design. Qilu University of Technology, 2013.6.8.

[4] Mu Yajun. Study on Packaging Design of Longjing Tea in West Lake. Hangzhou Normal University, 2013. 10. 8.

[5] Wang Jiahui. On the Extension Function of Packaging Design. Southwest Jiaotong University, 2012.9.29.

[6] Lin Yuehua, Jiang Yu, Wu Yanye. Research into the Difference between Online Shopping Packaging and Traditional Packaging. Packaging World, 2016.6.10-12. 
[7] Liao Yuyao, Chen Danqing, Li Wei, etc. Intelligent Packaging Research and Application Progress. Green Packaging, 2016.10, 39-46.

[8] Xu Wenkai. The New Darling of Smart Packaging-RF Technology [J]. Printing Magazine, 2013, (02):57-59.

[9] Hill SC, Htet Y, Kauffman J, Han IY, Dawson PL, Pennington WT, Hanks TW. Polydiacetylene-based smart packaging [J]. Phys Meth Food Anal: Am Chem Soc, 2013, 1138: 137-54.

[10] Verghese K. Crossin E, JoHands M. Packaging Materials [M]. Pack- aging for Sustainability. Springer London: 2012. 